\title{
REMARKS ON THE CLASSICAL INVERSION FORMULA FOR THE LAPLACE INTEGRAL
}

D. V. WIDDER AND N. WIENER

If a function $f(s)=f(\sigma+i \tau)$ is defined for $\sigma>0$ by the Iaplace integral

$$
f(s)=\int_{0}^{\infty} e^{-s t} \phi(t) d t
$$

then the classical inversion formula is

$$
\phi(t)=\frac{1}{2 \pi i} \int_{c-i \infty}^{c+i \infty} f(s) e^{s t} d s, \quad c>0, t>0 .
$$

Conditions for the validity of this formula have frequently been discussed. However, the authors know of no adequate treatment* of the case when $\phi(t)$ belongs to $L^{2}$ in $(0, \infty)$ :

$$
\int_{0}^{\infty}|\phi(t)|^{2} d t<\infty \text {. }
$$

We employ here the usual notation,

$$
\underset{a \rightarrow \infty}{\lim } . \phi_{a}(t)=\phi(t)
$$

to mean that $\phi_{a}(t)$ and $\phi(t)$ belong to $L^{2}$ in $(-\infty, \infty)$ and that

$$
\lim _{a \rightarrow \infty} \int_{-\infty}^{\infty}\left|\phi_{a}(t)-\phi(t)\right|^{2} d t=0 .
$$

It is clear first that if (3) holds then (1) converges absolutely for $\sigma>0$, since

$$
|f(\sigma+i \tau)|^{2}=\left|\int_{0}^{\infty} e^{-s t} \phi(t) d t\right|^{2} \leqq \int_{0}^{\infty} e^{-2 \sigma t} d t \int_{0}^{\infty}|\phi(t)|^{2} d t .
$$

Moreover, by the Plancherel theorem regarding Fourier transforms,

$$
\underset{a \rightarrow \infty}{\lim } \cdot \int_{0}^{a} e^{-i \tau t} \phi(t) d t
$$

* But compare G. Doetsch, Bedingungen filr die Darstellbarkeit einer Funktion als Laplace Integral und eine Umkehrformel fïr die Laplace-Transformation, Mathematische Zeitschrift, vol. 42 (1936), p. 272, Theorem 1. 
exists. We denote it by $f(i \tau)$. The same theorem gives us at once that

$$
\underset{a \rightarrow \infty}{\lim .} \frac{1}{2 \pi} \int_{-a}^{a} f(i \tau) e^{i \tau t} d \tau=\left\{\begin{array}{cc}
\phi(t), & t>0 \\
0, & t<0
\end{array}\right.
$$

or

$$
\underset{a \rightarrow \infty}{\lim . \mathrm{l}} \frac{1}{2 \pi i} \int_{-i a}^{i a} f(s) e^{s t} d s=\left\{\begin{array}{cc}
\phi(t), & t>0, \\
0, & t<0 .
\end{array}\right.
$$

Hence (2) with $c=0$ is valid in the sense of (4). However, if $c>0$, (2) is no longer valid in this sense.

If $c>0$, it is again clear from the Plancherel theorem that

$$
\underset{a \rightarrow \infty}{\lim .} \frac{1}{2 \pi} \int_{-a}^{a} f(c+i \tau) e^{i \tau t} d \tau=\left\{\begin{array}{cc}
e^{-c t} \phi(t), & t>0 \\
0, & t<0 .
\end{array}\right.
$$

But this does not imply that

$$
\text { l.i.m. } \int_{a \rightarrow i a}^{c+i a} f(s) e^{s t} d s=\left\{\begin{array}{cc}
\phi(t), & t>0, \\
0, & t<0,
\end{array}\right.
$$

unless $f(s)$ is identically zero. For, set $\phi_{a}(t)=\int_{-a}^{a} f(c+i \tau) e^{i r t} d \tau$. It will be sufficient to show that

$$
\int_{-\infty}^{\infty} e^{2 c t}\left|\phi_{a}(t)\right|^{2} d t=\infty
$$

for some $a$. Choose $a$ so that

$$
f(c+i a) \neq f(c-i a) .
$$

This is possible, for otherwise we should have by use of (1) that

$$
\int_{0}^{\infty} e^{-c t} \phi(t) \sin a t d t=0
$$

for all $a$. By the uniqueness theorem for the Fourier sine transform this would imply that $\phi(t)$ is equivalent to zero and that $f(s)$ is identically zero. In fact we see that (6) may be satisfied for some $a$ in every interval however small.

An integration by parts of the integral defining $\phi_{a}(t)$ gives

$$
\begin{aligned}
\phi_{a}(t) & =\frac{f(c+i a) e^{i a t}-f(c-i a) e^{-i a t}}{i t}-\frac{1}{t} \int_{-a}^{a} e^{i \tau t} f^{\prime}(c+i \tau) d \tau \\
& =\frac{f(c+i a) e^{i a t}-f(c-i a) e^{-i a t}}{i t}+o\left(\frac{1}{|t|}\right), \quad|t| \rightarrow \infty,
\end{aligned}
$$




$$
\begin{aligned}
& =\frac{e^{i a t}}{i t}\left[\{f(c+i a)-f(c-i a)\}+f(c-i a)\left(1-e^{-2 i a t}\right)\right] \\
& \quad+o\left(\frac{1}{|t|}\right) .
\end{aligned}
$$

Hence

$$
t^{2}\left|\phi_{a}(t)\right|^{2} \geqq\left[\frac{k}{2}-2 l|\sin a t|\right]^{2}, \quad t>t_{0},
$$

where $t_{0}$ is a sufficiently large positive number and

$$
k=|f(c+i a)-f(c-i a)| \neq 0, \quad l=|f(c-i a)| .
$$

Since $2 l|\sin a t|<k / 4$ in an interval of length $\delta$, say, about $t=0$ and in intervals congruent to this one, modulo $\pi / a$, it is clear that the integrand of (5) exceeds $k^{2} e^{2 c t} / 16 t^{2}$ in infinitely many intervals of length $\delta$. This is sufficient to insure the divergence of the integral.

We collect our results in the following form:

THEOREM. If $\phi(t)$ belongs to $L^{2}$ in $(0, \infty)$, then it has a Laplace transform $f(\sigma+i \tau)$ defined for $\sigma>0$ by the absolutely convergent integral

$$
f(\sigma+i \tau)=\int_{0}^{\infty} e^{-(\sigma+i \tau) t} \phi(t) d t
$$

and for $\sigma=0$ by

$$
f(i \tau)=\underset{a \rightarrow \infty}{\operatorname{li} . \mathrm{m}} \cdot \int_{0}^{a} e^{-i \tau t} \phi(t) d t .
$$

The inversion formula

$$
\underset{a \rightarrow \infty}{\operatorname{iim}} \cdot \frac{1}{2 \pi i} \int_{c-i a}^{c+i a} f(s) e^{s t} d s=\left\{\begin{array}{cl}
\phi(t), & t>0, \\
0, & t<0,
\end{array}\right.
$$

is false $(f(s) \not \equiv 0)$ for $c>0$ and valid for $c=0$. For all $c \geqq 0$

$$
\underset{a \rightarrow \infty}{\lim } \cdot \frac{1}{2 \pi} \int_{-a}^{a} f(c+i \tau) e^{i \tau t} d \tau=\left\{\begin{array}{cc}
e^{-c t} \phi(t), & t>0, \\
0, & t<0 .
\end{array}\right.
$$

HARVARD UNIVERSITY AND

Massachusetts Institute of Technology 